\title{
Index may show outcome differences in unilateral cleft lip and palate patients at 5 -years of age
}

\author{
Atack N E, Hathorn I, Dowell T, Sandy J, Semb G, Leach A. Early detection of differences in surgical outcome for \\ cleft lip and palate. BrJ Ortho. 1998; 25; 181-185.
}

Objective To examine whether differences in dental arch relationships of 5-year-old children born with a unilateral cleft lip and palate could be detected.

Materials and Methods 40 sets of study models from each of two surgical units (Bristol \& Oslo) were assessed using the 5year old index. Models from each unit were assessed twice on one day at least 1 week apart by two examiners. Positions of the models in the series were reallocated using randomly generated numbers to minimise the influence of memory. Inter and intraexaminer variability was assessed using weighted kappa statistic.

Results Intra-examiner variability was good to very good while inter-examiner variability was moderate to good. Both initial and subsequent examinations of the models by the examiners showed that the Oslo sample had a greater number of patients categories 1 and 2 which are considered to indicate better long term growth outcomes. Other differences were also detected between the two modelled samples suggesting that it is possible to detect differences in surgical outcomes at 5-years of age.

Conclusions Differences in dental arch relationships in patients with unilateral CLP are apparent at 5 years of age and the use of this index appears to show difference in outcomes by which it may be feasible to compare outcomes from different surgeons.
Address for reprints: Dr Hathorn, Bristol Dental Hospital, Lower Maudlin Street, Bristol, BS1 2LY, UK

\section{Commentary}

Differences in surgical outcomes for unilateral CLP patients are difficult to study due to high variability in treatment approaches and patient characteristics. Complicating the problem further is the long interval between the surgical procedure (first year of life) and the final evaluation point (post-adolescence). Therefore, the proposal by Atack et al. using a straightforward index of dental arch relationship may provide early outcome information at age 5 is intriguing.

In previous papers Atack and co-workers described a rating system for evaluation of dental study models obtained from unilateral cleft lip and palate patients. ${ }^{1,2}$ The method has high intrarater reliability and reproducibility with moderate agreement between raters. They determined that, in most cases, dental relationships stayed the same or became worse between 5 and 10 years. ${ }^{1}$ However, they were not able to predict on an individual basis the need for osteotomy at age 16-18 from information obtained from the 5 year dental casts. ${ }^{2}$

In this paper, dental relationships were compared from study models of unilateral CLP patients seen in two centers, Bristol and Oslo. Significant differences in the proportion of good to poor dental arch relationships were related to the geographic location of the patients.

At this point, it is tempting to relate the differences observed to surgical care alone. However, other factors could contribute to the results, particularly since little information was given about the samples other than that the gender mix was similar for both groups. Theoretically, variability in factors such as the following could influence the outcome also: severity of cleft before repair, other medical findings, facial type due to race or ethnicity, mandibular growth vector, timing of palatal repair, experience of the surgeon, patient dropout, medical referral patterns, etc. Further studies may clarify the roles of other factors.

The reason for choosing an opinion, the need for orthognathic surgery, as the criterion for validation was not explained adequately in the paper. Rates of utilisation of surgical procedures are highly linked to the training of the surgeon - insurance coverage, etc which vary with geography. More quantitative criteria could be considered, such as actual measurements of facial appearance, skeletal and dental disharmony, patient satisfaction, or function.

Longitudinal research is extremely difficult to conduct and interpret, especially when randomisation is not realistic for inclusion in study design. In that situation, carefully monitored case studies interpreted with caution can add to our knowledge, guide clinicians, and benefit patients.

1 Atack, N E, Hathorne, I S, Semb, G, Dowell, $T$ and Sandy, J R. A new Index for assessing surgical outcome in unilateral cleft lip and palate subjects aged 5 - reproducibility and reliability, Cleft Palate Craniofacial) 1997; 34: 242-246.

2 Atack, NE, Hathorne, IS, Mars, M and Sandy, I R The use of study models to assess surgical outcome in cleft lip and palate subjects at the age of 5 . Euro I Ortho, 1997; 19: 165-170.

Professor Carla A. Evans, Department of Orthodontics, College of Dentistry,

University of Illinois at Chicago, USA 